\title{
Étude de la calcination du phosphate clair de youssoufia (Maroc)
}

\author{
El mokhtar El OUARDI
}

Laboratoire de mécanique et énergétique, Département de physique, Faculté des

Sciences, BP 20, El Jadida, Maroc

*Correspondance,courriel : m.elovardi@yahoo.fr

\section{Résumé}

Dans cet article, nous avons étudié le temps et la température de calcination du phosphate sédimentaire de Youssoufia. Notre objectif est d'optimiser les dépenses liées à l'énergie destinée à brôler les matières organiques et d'obtenir le point qualité satisfaisant les exigences de son utilisation. La calcination a été effectuée dans un four réglable en température suivi d'une extinction et d'un tamisage. Nous avons pu augmenter la teneur en Phosphates de chaux des os (BPL) de $68 \%$ à $74.8 \%$ et diminuer le carbone organique $\left(\mathrm{C}_{\text {org }}\right)$ et le dioxyde de carbone $\left(\mathrm{CO}_{2}\right)$ respectivement de $1.8 \%$ à 0.15 $\%$ et de $6.8 \%$ à $1.8 \%$. De plus, nous avons constaté que la distribution granulométrique du phosphate calciné varie en fonction de la variation de la vitesse de son refroidissement.

Mots-clés : Phosphate, matière organique, calcination, Youssoufia, Maroc

\section{Abstract \\ Study of the calcination of phosphates clear of youssoufia (Morocco)}

In this work, we studied the time and the temperature of calcination of sedimentary phosphate of Youssoufia. Our aim is the optimization of the expenditure related to energy distinated to burn the organic matter and therefore obtain a point quality suitable to the requirements of its use. The calcination was carried out in an adjustable furnace in temperature followed by extinction and a sifting. The results show that we can increase the content of Bone Phosphate of Lame (BPL) from $68 \%$ to $74.8 \%$ and decrease the organic carbon and the carbon dioxide respectively of $1.8 \%$ to 0.15 and $6.8 \%$ to $1.8 \%$. 
Moreover, we note that the granulometric distribution of calcined phosphates varies according to the rate of its cooling.

Keywords : Phosphate, organic matter, calcination, Youssoufia, Morroco

\section{Introduction}

Les gisements sédimentaires de phosphates naturels sont composés principalement d'apatite de formule générale: $M_{10}\left(Z O_{4}\right)_{6} X_{2}$ avec pour cations les plus communs : $M=C a$, $\mathrm{Sr}, \ldots ; Z=\mathrm{P}, \mathrm{As}, \mathrm{Si}, \ldots ; \mathrm{X}=\mathrm{F}, \mathrm{OH}, \mathrm{Cl} \ldots$.. Ils sont représentés surtout par les pôles fluorapatite-hydroxylée $\left[\mathrm{Ca}_{5}\left(\mathrm{PO}_{4}\right)_{3}(\mathrm{OH}, \mathrm{F})\right]$ et/ou la fluorapatite carbonatée (appelée francolite) $\left[\mathrm{Ca}_{5}\left(\mathrm{PO}_{4}, \mathrm{CO}_{3}, \mathrm{OH}\right)_{3}(\mathrm{OH}, \mathrm{F})\right][\mathrm{l}]$. Ces gisements sédimentaires peuvent contenir des apatites ayant une gamme très large de propriétés physiques et des caractéristiques chimiques et cristallographiques très variées, en raison des conditions géologiques et des altérations après dépôt [2].

En plus de la matrice principale contenant le phosphore, les gisements phosphatés contiennent des minéraux accessoires ou des impuretés de la gangue qui se différencient selon les conditions de sédimentation. Ces impuretés sont parfois géologiquement bien isolées sous forme de couches bien séparées ou intimement mélangées au minerai. Elles incluent, dans diverses combinaisons et concentrations, de la matière organique, de la silice, des minéraux argileux, de la calcite, de la dolomie et des oxydes hydratés de fer et d'aluminium [1,3]. Elles ont une influence sur les processus de valorisation des phosphates et sur l'efficacité du phosphate naturel utilisé en application directe [1,3].

La connaissance au préalable de la nature des stériles contenues dans le minerai phosphaté est très importante parce qu'elle permet de déterminer exactement le type de traitement approprié à ce minerai. En fonction de sa teneur en BPL, le minerai peut être classé en quatre catégories : le phosphate très basse teneur (TBT), le phosphate basse teneur (BT), le phosphate moyen teneur (MT) et le phosphate haute teneur (HT). Les phosphates HT et MT ont des teneurs en BPL supérieures à $68 \%$ et sont considérés comme des produits marchands, pour lesquels on utilise des méthodes conventionnelles telles que le séchage, ou quelque autres techniques de séparation physique.

Les différents aspects du traitement thermique ont été étudiés par de nombreux auteurs [4-7]. Par contre, pour les phosphates BT et TBT, quelques autres techniques spéciales peuvent être aussi appliquées telles que la calcination, dont l'un des effets essentiels est l'élimination d'une partie de la matière organique sous forme gazeuse $\left(\mathrm{CO}_{2}, \mathrm{H}_{2} \mathrm{O} \ldots\right)$. Elle agit également sur les propriétés d'usage (composition, granulométrie, densité, 
porosité, surface spécifique, dureté, capacité calorifique, conductivité thermique....) [8-13].

Du point de vue énergétique, la matière organique donne un pouvoir calorifique important. Mais sa présence dans le phosphate exige habituellement un ajout supplémentaire de l'acide sulfurique pendant la fabrication d'acide phosphorique. L'élimination de la matière organique est importante parce qu'elle constitue un élément gênant qui réduit les sulfates en sulfures entraînant ainsi une intense corrosion des réacteurs lors de la fabrication de l'acide phosphorique [12]. En outre, Elle provoque aussi la formation des mousses lors de l'attaque par l'acide sulfurique. Ce qui défavorise le refroidissement de la réaction, ainsi que l'augmentation de la température et de la viscosité, ce qui conduit à la formation des phosphogypses hemihydrate. Il en résulte une mauvaise filtration qui affecte la qualité de l'acide phosphorique et fait diminuer le rendement chimique $[7,12]$. D'ailleurs, le dioxyde du carbone contamine aussi le matériel en donnant une couleur noire à l'acide produit et contribue à la pollution de l'environnement.

Plusieurs difficultés dues à la diversité des propriétés physiques et des caractéristiques chimiques et cristallographiques, surviennent lorsqu'on étudie les phosphates comme complexe. Ces difficultés sont liées fortement à la présence du composant organique. Aujourd'hui, il n'existe aucune technique conduisant à une séparation complète des impuretés des apatites sans modification de l'un d'eux. Le point de départ de valorisation des phosphates est le traitement mécanique et thermique pour lesquels la calcination demande une énergie importante et donc une quantité de combustible élevée, ce qui augmente le coût de traitement et pollue l'environnement.

Dans ce travail, nous nous intéresserons à l'optimisation de traitement thermique en déterminant le temps de séjour et la température de calcination du minerai phosphaté de la zone d'étude, de granulométrie comprise entre $40 \mu \mathrm{m}$ et $500 \mu \mathrm{m}$, tout en minimisant la matière organique $(\% \mathrm{C})$ et les carbonates $\left(\% \mathrm{CO}_{2}\right)$ et en augmentant la teneur en $\mathrm{BPL}$ en ratio avec la teneur de la chaux.

\section{Zone d'étude}

Le Maroc dispose des trois quarts de la réserve mondiale en minerais phosphatés et occupe la première place dans l'exportation. Les séries sédimentaires phosphatées marocaines recèlent des quantités relativement importantes de minéraux associés [14]. Les principaux faciès impliqués dans cette étude sont des phosphates sableux granulaires, des calcaires et dolomies, des marnes, argiles et silicifications diagénétiques intercalées. En terme de microfaciès, les phosphorites sont toujours des 
composites d'origine diversifiée (autochtone et/ou allochtone) [15]. Ces propriétés générales ont été décrites par L. Bilali et coll. [9]. Les gisements de phosphates du Maroc se localisent dans un certain nombre de bassins situés dans quatre domaines géographiques différents. Pour réaliser notre objectif, nous nous sommes intéressés à l'étude des phosphates issus d'un nouveau gisement de la mine de Bouchane, située à 40 $\mathrm{km}$ du site de Youssoufia (Bassin de Gantour) (Figure ).

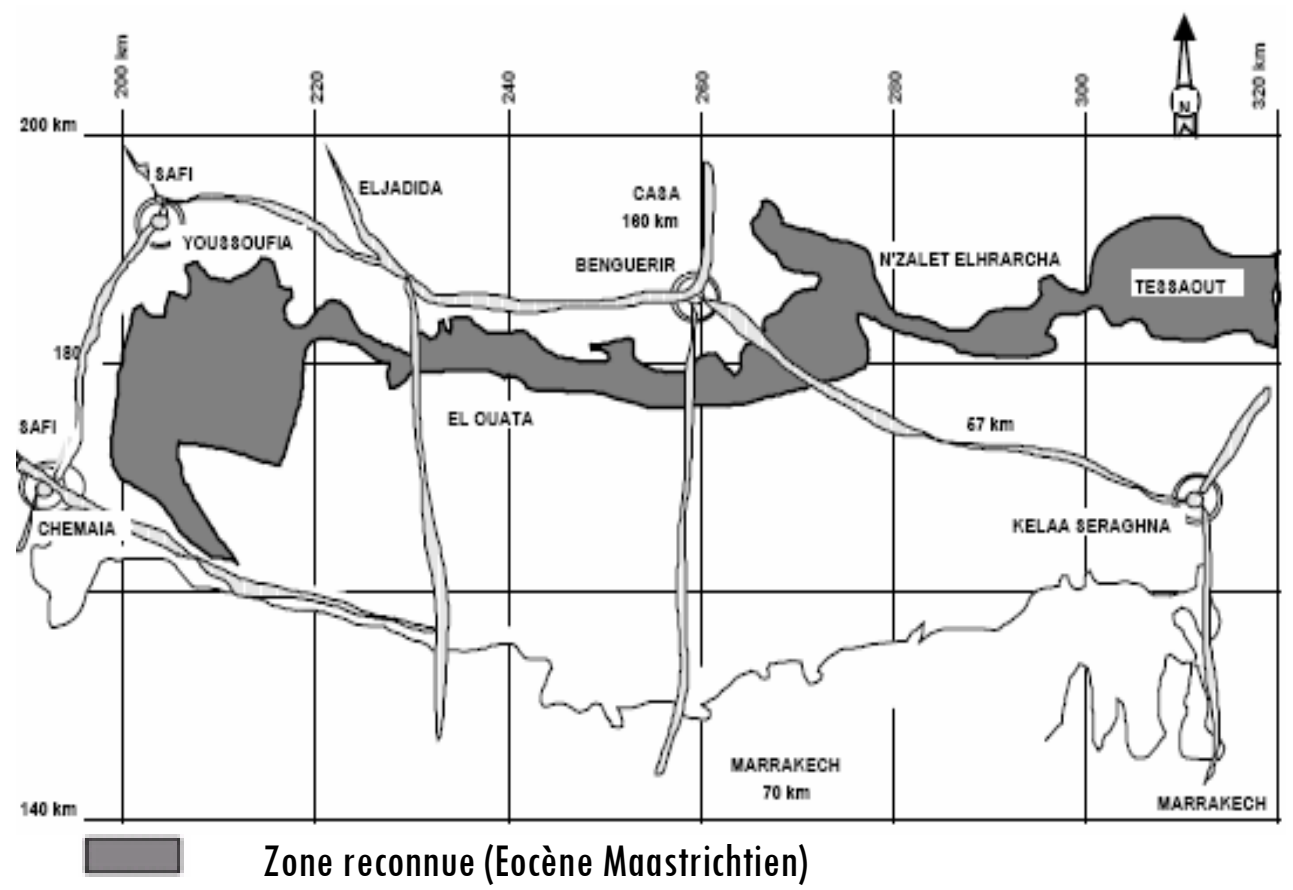

Figure 1 : Gisement de Gantour (Youssoufia \& Benquerir)

\section{Caractérisation physicochimique du phosphate brut de la zone étudie}

La composition chimique et la granulométrie déterminent la réactivité de phosphate naturel. La répartition granulométrique a été réalisée par tamisage manuel, à voie sèche avec une série de tamis de toiles en Nylon de maille à trou ronds en progression géométrique. Le Tableau $I$ illustre la distribution granulométrique de site de phosphate de la zone étudie. Ainsi que la composition de leurs matières minérales et organique représentée dans le Tableau 2. 
Tableau 1. Distribution granulométrique avant la classification

\begin{tabular}{|l|l|l|l|l|l|l|l|l|l|l|l|l|l|}
\hline $\begin{array}{l}\text { Maille } \\
(\mu \mathrm{m})\end{array}$ & 2000 & 1250 & 1000 & 800 & 500 & 315 & 250 & 200 & 160 & 125 & 80 & 40 & $<40$ \\
\hline $\begin{array}{l}\text { Refus } \\
(\%)\end{array}$ & 3.32 & 2.68 & 1.61 & 7.18 & 26.83 & 15.94 & 10.66 & 10.83 & 7.88 & 7.75 & 2.27 & 1.9 & 1.12 \\
\hline
\end{tabular}

Tableau 2 : Composition chimique moyenne du minerai de phosphate du Youssoufia avant la calcination

\begin{tabular}{|c|c|c|c|c|}
\hline BPL (\%) & $\mathrm{H}_{2} \mathrm{O}(\%)$ & $\mathrm{C}_{\text {org }}(\%)$ & $\mathrm{CO}_{2}(\%)$ & Autres $(\%)$ \\
\hline 68 & 14 & 1.8 & 6.8 & 9.4 \\
\hline
\end{tabular}

L'analyse minéralogique a été effectué à l'aide d'un diffractomètre XPRT MPD PanalyticalPhilips, à anticathode de cuivre $(\lambda K \alpha=1,5405 \mathrm{~A})$. Le diffractogramme (Figure 2) révèle la présence des phases suivantes : la fluorapatite $\mathrm{Ca}_{10}\left(\mathrm{PO}_{4}\right)_{6} \mathrm{~F}_{2}(2 \theta: 32,173$; $33,280 ; 49,786 ; 64,179 ; 40,227 ; 34,331 ; 25,879)$, le quartz $\mathrm{SiO}_{2}(2 \theta: 26,587 ; 67,861$; $50,079 ; 20,885)$, et les carbonates qui sont sous forme de la dolomite $\mathrm{CaMg}\left(\mathrm{CO}_{3}\right)_{2}(2 \theta$ : $30,917 ; 50,674 ; 41,187)$ et de la calcite $\mathrm{CaCO}_{3} .(2 \theta: 45,790 ; 26,189 ; 48,376 ; 33,153$; $38,101 ; 36,041 ; 27,081 ; 52,553)$. La variation du nombre des raies citées est due à la complexité du phosphate.

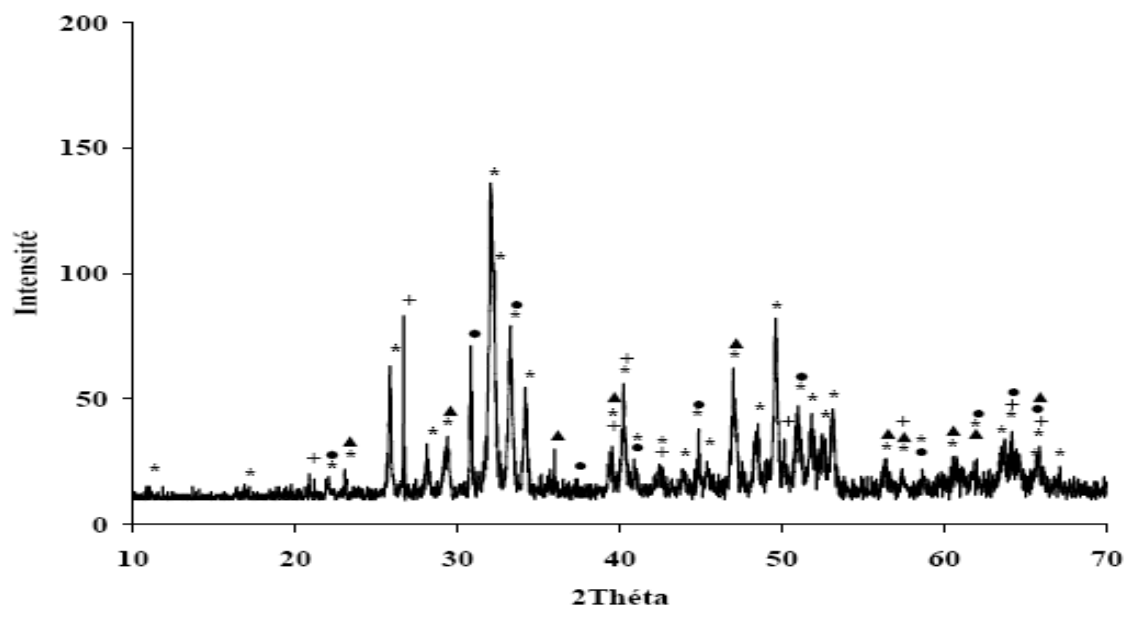

Figure 2 : Diffractogramme $R X d u$ phosphate brut 


\section{Procédure Expérimentale}

Les essais sont effectués à l'échelle du laboratoire, dans un four réglable en température, sur le phosphate de Youssoufia, de granulométrie comprise entre $40 \mu \mathrm{m}$ et $500 \mu \mathrm{m}$. La classification se fait en réduisant les gros grâce à un broyeur à marteau par des opérations successives portant sur les refus à la maille de $500 \mu \mathrm{m}$ et un tamisage des fines inférieures à $40 \mu \mathrm{m}$. Ainsi, on ne récupère que la poudre de granulométrie comprise entre $40 \mu \mathrm{m}$ et $500 \mu \mathrm{m}$ riche en phosphore $[7,11]$. Les résultats de la classification sont présentés dans le Tableau 3.

Tableau 3 : Distribution granulométrique après la classification

\begin{tabular}{|l|l|l|l|l|l|l|l|}
\hline $\begin{array}{l}\text { Maille } \\
(\square \mathrm{m})\end{array}$ & 315 & 250 & 200 & 160 & 125 & 80 & 40 \\
\hline $\begin{array}{l}\text { Refus } \\
(\%)\end{array}$ & 46.06 & 18.25 & 12.5 & 9.25 & 7.64 & 3.3 & 3.0 \\
\hline
\end{tabular}

L'étude du temps de calcination est réalisée en introduisant des échantillons de phosphate dans le four, pour une durée de 40 minutes, à une température fixe $1600^{\circ} \mathrm{C}$, $650^{\circ} \mathrm{C}, 700^{\circ} \mathrm{C}, 750^{\circ} \mathrm{C}, 800^{\circ} \mathrm{C}, 850^{\circ} \mathrm{C}$ et $\left.900^{\circ} \mathrm{C}\right)$. Pour chaque température et à chaque cinq minutes, un échantillon est prélevé pour analyser les compositions chimiques de l'apatite $\left(\mathrm{C}_{\text {org' }} \mathrm{CO}_{2}\right.$, $\mathrm{BPL}$ et $\left.\mathrm{CaO}\right)$. La composition chimique de l'échantillon brut a été déterminée par volumétrie. Le taux d'humidité est déterminé par un analyseur d'humidité MRS 120-3.

La température optimale de calcination est déterminée par l'étude de la variation de la composition chimique de ses différentes espèces $\left(\mathrm{C}_{\text {org }}, \mathrm{CO}_{2}, \mathrm{BPL}, \mathrm{CaO}\right)$ en fonction de la température $\left(600^{\circ} \mathrm{C}<\mathrm{T}<900^{\circ} \mathrm{C}\right)$. En plaçant sept $(7)$ échantillons dans un four et à chaque augmentation de $50^{\circ} \mathrm{C}$ de la température, un échantillon est prélevé pour analyser la composition chimique de l'apatite $\left(\mathrm{C}_{\text {org' }}, \mathrm{C}_{2}, \mathrm{BPL}, \mathrm{CaO}\right)$.

Lors de cette étude de la température optimale de calcination et le temps de séjour, on vérifie qu'il n'y a pas de perte en masse de l'apatite riche en phosphore en aérant les autres échantillons par injection de l'air d'une façon nominale.

Avant l'analyse des compositions $\mathrm{C}_{\text {org' }} \mathrm{CO}_{2}, \mathrm{BPL}$ et $\mathrm{CaO}$, le produit calciné se refroidit sous la pression atmosphérique. 0 n procède par la suite à l'extinction et le tamisage pour éliminer la matière volatile adsorbée et la fraction granulométrique inférieur à $40 \mu \mathrm{m}$ qui est pauvre en phosphore. 


\section{Résultats}

Le protocole expérimental décrit précédemment nous a permis d'étudier la variation de la composition en $\mathrm{C}_{\text {org' }} \mathrm{CO}_{2}, \mathrm{BPL}$ et $\mathrm{CaO}$ en fonction de la température. Les résultats sont illustrés dans les Figures $3 a, 3 b, 3 c$ et $3 d$.

La figure $3 a$ montre que la teneur en carbone organique reste quasi-constante à sa concentration initiale $(1.8 \%)$ lorsque la température est comprise entre $600^{\circ} \mathrm{C}$ et $700^{\circ} \mathrm{C}$. De $700^{\circ} \mathrm{C}$ à $800^{\circ} \mathrm{C}$, la teneur en carbone commence à diminuer et au-delà de $800^{\circ} \mathrm{C}$, elle varie très faiblement et redevient sensiblement constante.

Le dioxyde de carbone commence à se dégager à partir de $600^{\circ} \mathrm{C}$ jusqu'à $800^{\circ} \mathrm{C}$. Au delà de $800^{\circ} \mathrm{C}$, la concentration de dioxyde de carbone reste relativement constante (Figure $3 b)$.

La teneur en BPL augmente de $600^{\circ} \mathrm{C}$ jusqu'à $800^{\circ} \mathrm{C}$ et au-delà de $800^{\circ} \mathrm{C}$, elle redevient sensiblement constante (Figure 3خ). Dans le même temps et d'une façon analogue comme l'indique la Figure $3 d$, la teneur en $\mathrm{Ca} 0$ varie de la même manière que celle de la BPL.

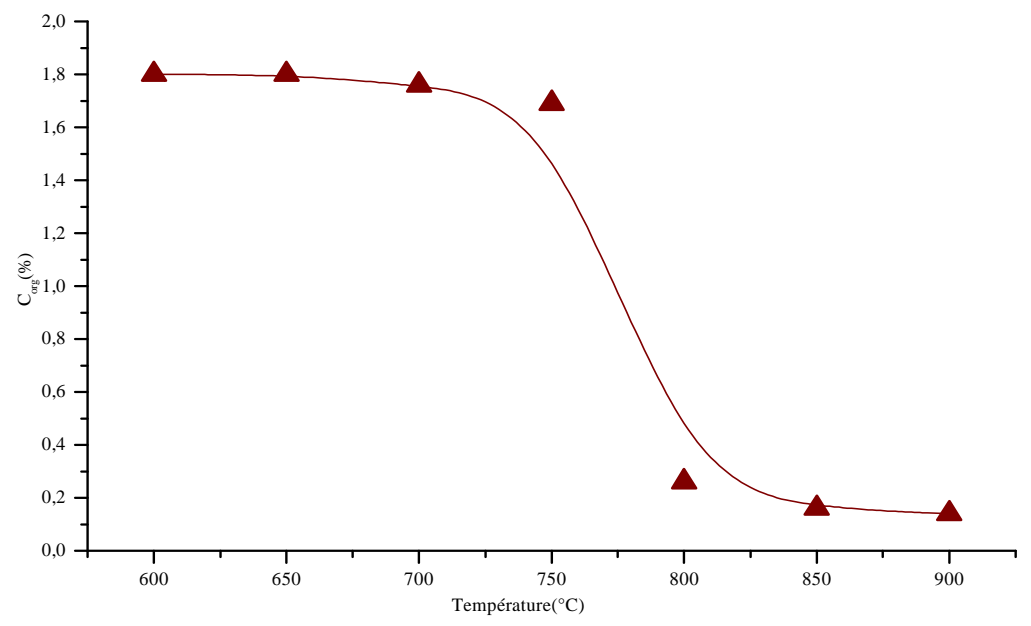

Figure 3a : Variation du $C_{\text {org }}$ en fonction de la température 


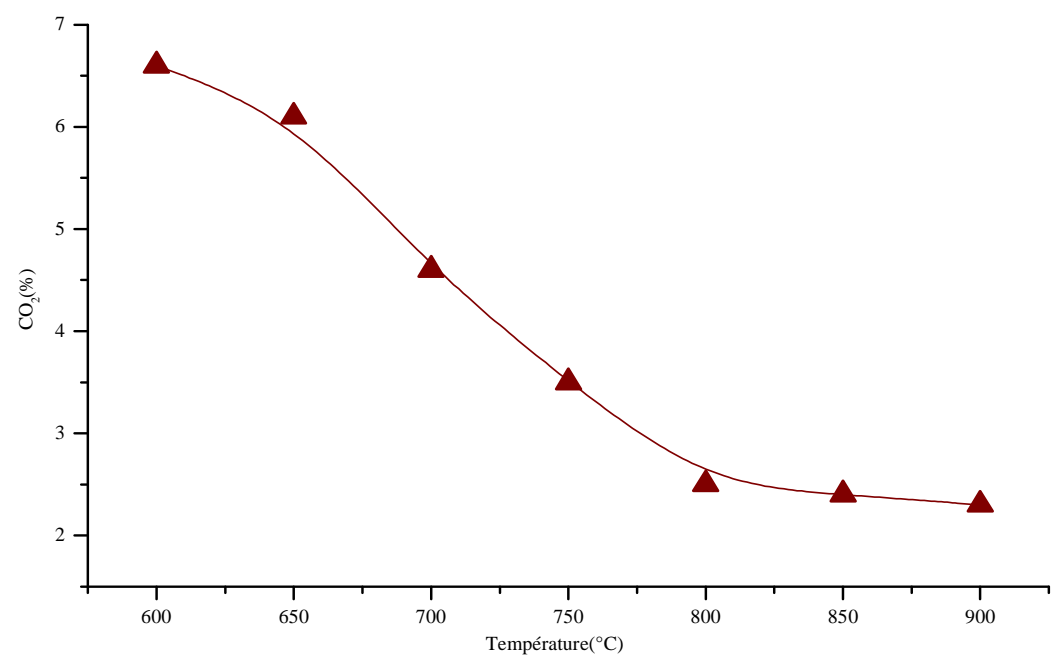

Figure $3 \mathrm{~b}$ : Variation de $\mathrm{CO}_{2}$ en fonction de la température

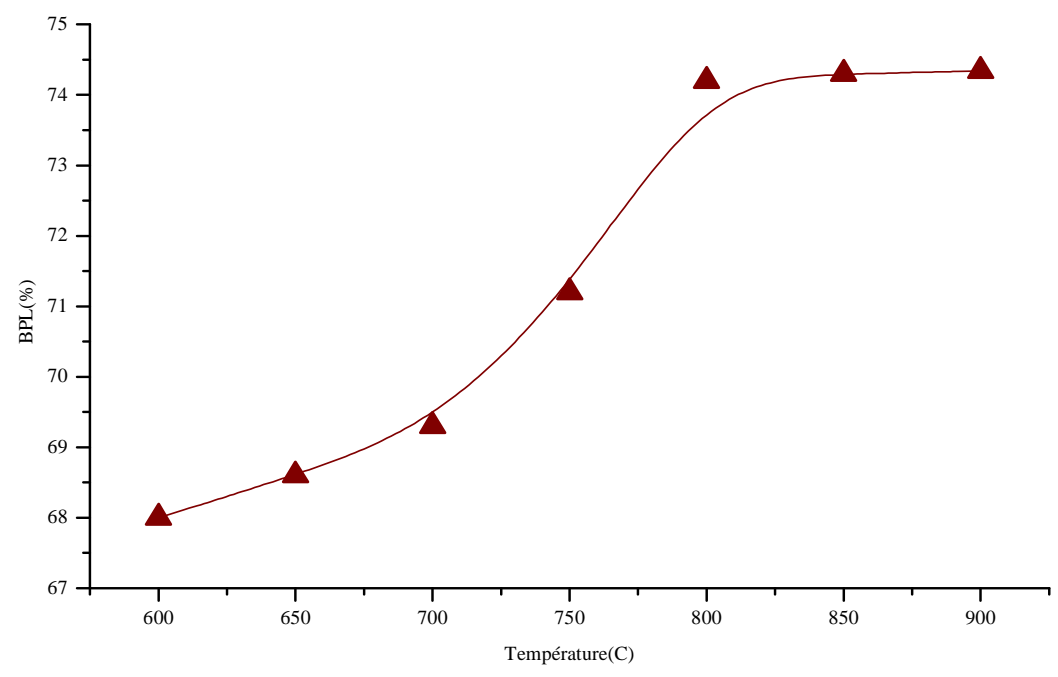

Figure 3c: Variation de BPL en fonction de la température 


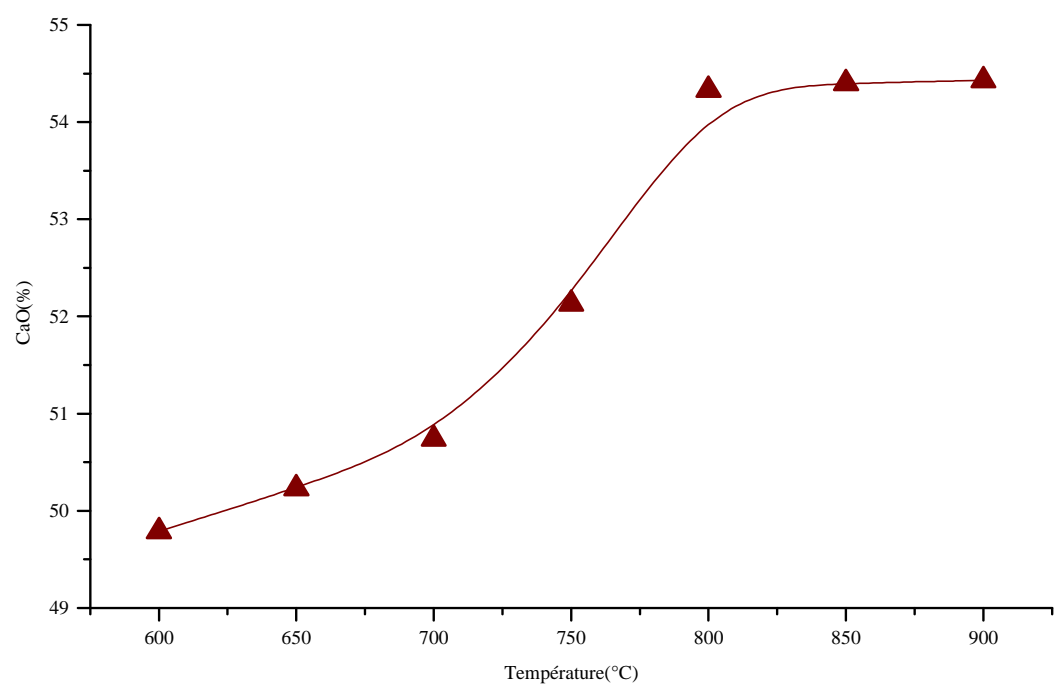

Figure 3d: Variation de CaO en fonction de la température

Par ailleurs, l'étude du temps de séjour de la calcination est très important dans toute optimisation de l'énergie destinée à la calcination. Les Figures $4 a, 4 b, 4 c$ et $4 d$ illustrent la variation de la composition en $\mathrm{C}_{\text {org }} \mathrm{CO}_{2}$, BPL et $\mathrm{CaO}$ en fonction du temps à différentes températures.

Comme nous l'avons vu précédemment, pour une température comprise entre $600^{\circ} \mathrm{C}$ et $700^{\circ} \mathrm{C}$, la teneur en carbone organique reste constante et à partir de $700^{\circ} \mathrm{C}$, le carbone organique commence a débiter en fonction du temps jusqu'à la température $800^{\circ} \mathrm{C}$ et un certain temps estimé à 30 minutes. Au-delà, il suit un profil de concentration quasiidentique (Figure 4a).

La Figure $4 b$ montre que la teneur de dioxyde de carbone diminue entre $600^{\circ} \mathrm{C}$ à $800^{\circ} \mathrm{C}$. Au delà de $800^{\circ} \mathrm{C}$ et un temps de 30 minutes, les courbes de la variation du dioxyde de carbone sont pratiquement confondues.

La teneur en BPL augmente jusqu'à environ $75 \%$ pendant 30 minutes puis cette concentration devient constante (Figure 4d). Dans le même temps, il apparaît que, la teneur en $\mathrm{Ca} 0$ augmente de la même manière que la teneur de BPL en fonction des temps et de la température (Figure 4 d). 

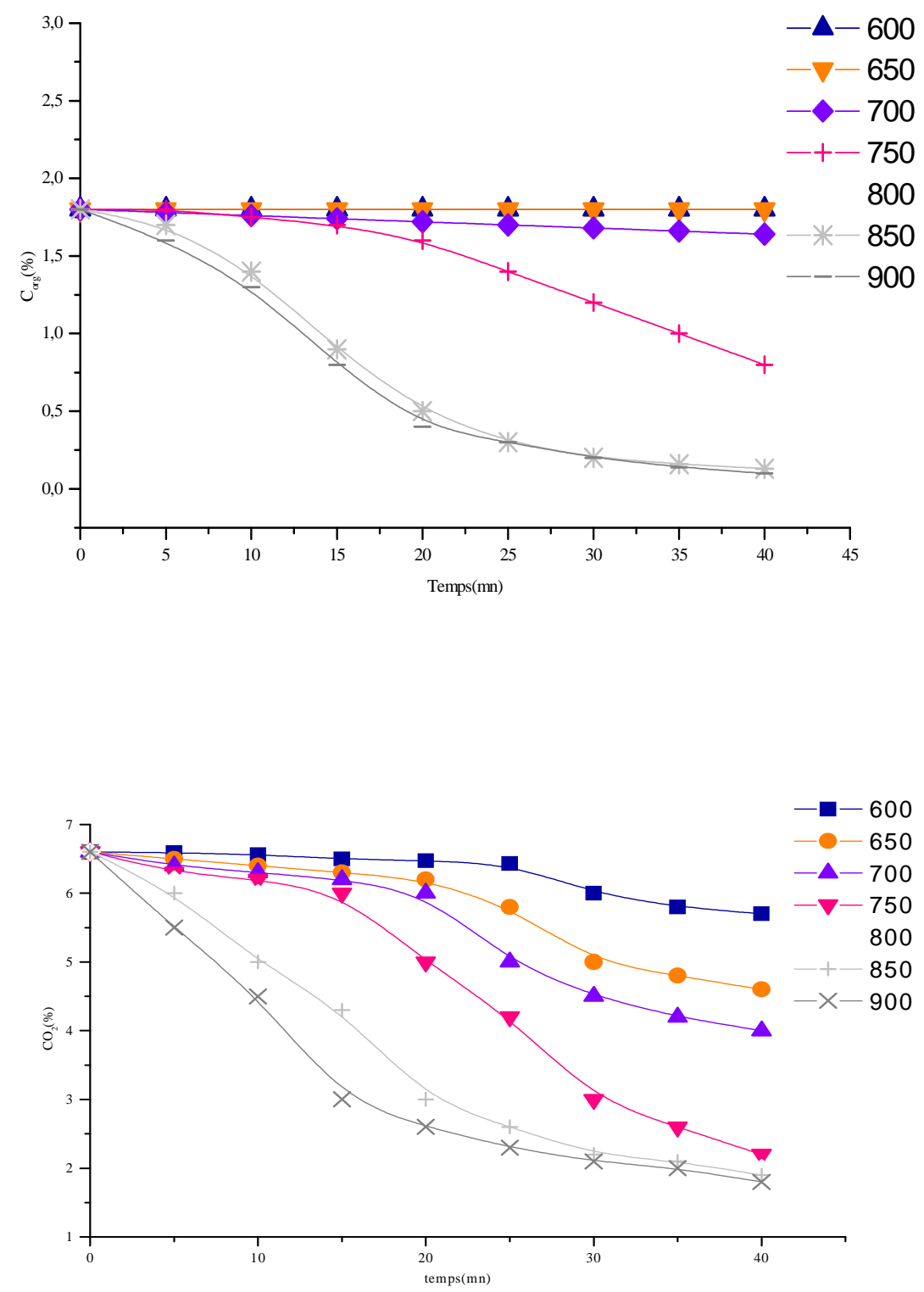

Figure 4b : Variation $\mathrm{CO}_{2}$ en fonction du temps entre $600^{\circ} \mathrm{C}$ et $900^{\circ} \mathrm{C}$ 


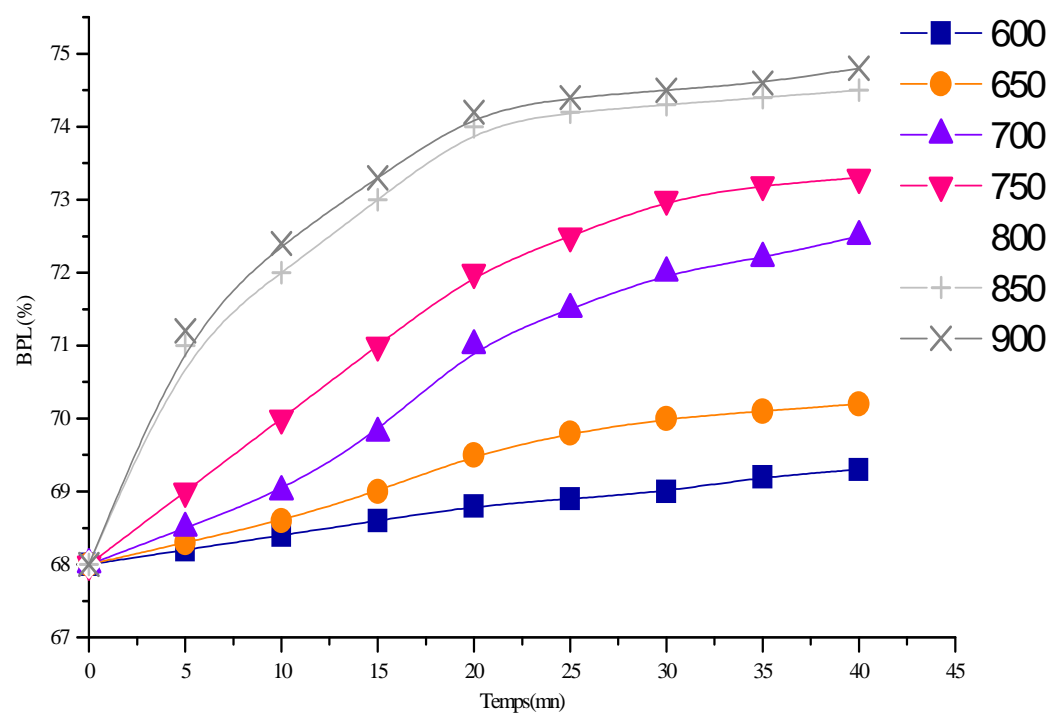

Figure 4c: Variation du BPL en fonction du temps entre $600^{\circ} \mathrm{C}$ et $900^{\circ} \mathrm{C}$

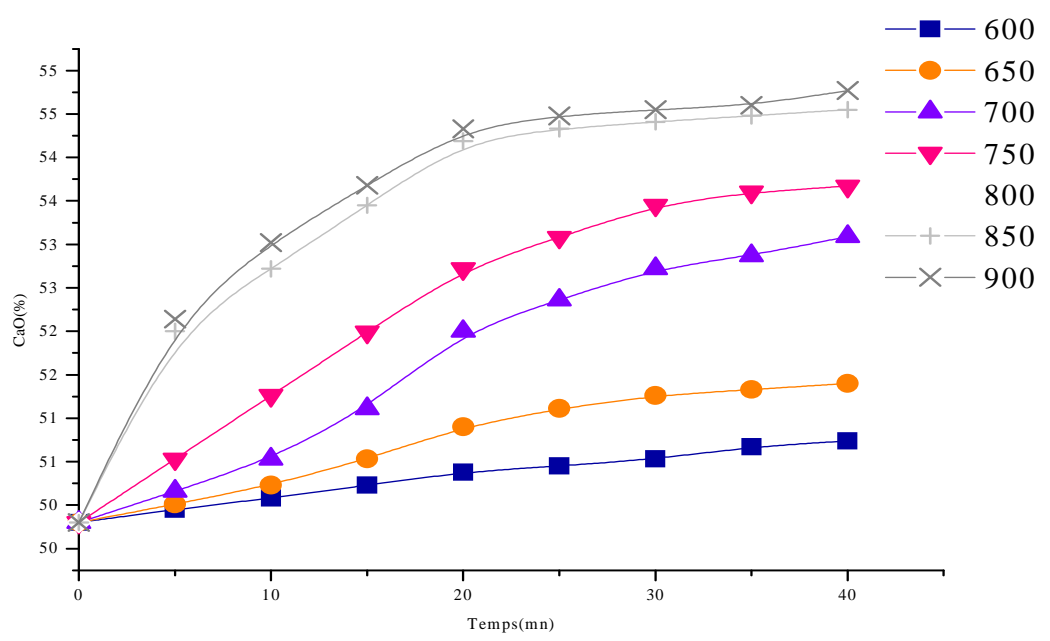

Figure 4d: Variation du CaO en fonction du temps entre $600^{\circ} \mathrm{C}$ et $900^{\circ} \mathrm{C}$ 


\section{Discussion}

Les différentes essais appliquées sur une série d'échantillon représentatif du phosphate de la zone étudie ont mis en évidence une étroite relation entre les phases minéralogiques, la répartition granulométrique et l'évolution des constituants principaux de contrôle dans la calcination en fonction du temps et de la température.

Il est important de noter que la présence de la matière organique et des carbonates se manifeste à travers de nombreux phénomènes qui se produisent au sein des différents constituants du phosphate, affectant les aspects thermocinétiques, les phénomènes thermoconvectifs et thermodiffusifs qui dépendent des propriétés thermophysiques du milieu (capacité calorifique, conductivité thermique, masse volumique, porosité, perméabilité,...) $[9,16]$. Ils jouent un rôle prépondérant sur les cinétiques des transformations et les phénomènes de transfert de chaleur et de masse ainsi que sur leurs couplages dans le milieu réactionnel lors du traitement thermique.

Les travaux antérieurs $[7,16,17]$ ont montré que la variation de la distribution granulométrique améliore les propriétés physiques surtout la surface spécifique et la porosité. Par conséquent, une exposition importante de la matière organique et des carbonates sur l'extérieur occasionne une grande surface spécifique du produit fini, et une diminution de la densité en fonction du temps et de la température du fait que la porosité contribue avec la masse volumique (densité) dans les lois de conservation de la matière. Ceci limite le taux de sédimentation de la pulpe du phosphate ou de la bouille lors de la production d'acide phosphorique avec l'absence du collage et de formation d'agrégats sur les parois du lit.

Les résultats obtenus dans cette étude montrent qu'il y'a des interactions entre les différents paramètres thermo-physico-chimiques. Le rôle joué par ces paramètres sur les mécanismes mis en jeu et sur l'obtention d'un produit de qualités meilleures est très important et suppose leur maîtrise. D'ailleurs, on ne peut pas négliger la différence entre les grandeurs thermophysiques réelles et apparentes.

\section{Conclusion}

Dans cette étude, nous avons établi dans les conditions optimales de la calcination en temps et en température. Le produit obtenu répond bien aux profils marchands et aux exigences d'utilisation sous les conditions d'une température d'environ de $800^{\circ} \mathrm{C}$ et un temps d'environ de 30 minutes avec une diminution de carbone organique à $91,67 \%$ et de dioxyde de carbone à $73,50 \%$ et une augmentation de BPL de $10 \%$. Le phosphate 
calciné contient un rapport de CaO/BPL permettant d'économiser la réaction de l'attaque du phosphate par l'acide sulfurique $\left(\mathrm{H}_{2} \mathrm{SO}_{4}\right)$. Ainsi, nous avons illustré l'intérêt de la prise en compte des couplages entre le processus de traitement et les propriétés physicochimiques.

\section{Références}

[1] - M. CUNEY, "Facteurs contrôlant la nature et la concentration des impuretés dans les phosphates sédimentaires", COVAPHOS I (ed) Proceedings of the first international conference on the valorization of phosphates and phosphorus compounds (2004) 11-14.

[2] - G. S. Michal, Chemie der Erde geochemistry, 64 (2004) 329-357.

[3] - United Nations Industrial Development Organization (UNIDO) \& IFDC. Fertilizer manual. Dordrecht, the Netherlands, Kluwer Academic Publishers (1998) 615 pp.

[4] - P. Blazy, J. C. Samama, C. R. Acad. Sci. Paris, série 333 (2001) 271-276.

[5] - J. L. Lacout, Ann. Chim. Sci Mat., 26 (6) (2001)1-3.

[6] - B. R. Stanmore, P. Gilot, Fuel Processing Technology (2005)

[7] - M. Ashraf, Z. I. Zafar, T. M. Ansari, J. of research(Science), Pakistan, Vol. 18, $\mathrm{N}^{\circ} 2$, (2007) 145-157.

[8] - K. Souhila, II Décomposition des matières organiques et stabilisation des métaux lourds dans les sédiments de dragage II, Instituł National des Sciences Appliquées de Lyon, Thèse (2005).

[9] - L. Bilali, M. Benchanaa et A. Mokhlisse, Ann. Chim. Sci. Mat. 25 (2000) 663-678.

[10] - L. Bilali, M. BenChanaa, A. Mokhlisse, J. Electromagnetic and Microwave Power 37(3) (2002) 145-166.

[11] - L. Bilali, A. Aouad, K. El Harfi, M. BenChanaa, A. Mokhlisse, Journal of analytical and. Applied. Pyrolysis 65 (2002) 221-237.

[12] - M. Khaddor, M. Ziyad, J. Joffre, A. Amblés, Chemical Geology, 186 (2002) 17-30.

[13] - L. Bilali, M. BenChanaa, K. El Harfi, A. Mokhlisse, A. outzourhit, J. Anal. Appl. Pyrolysis 73 (2005) 1-15.

[14] - L. Bilali, M. Kouhila, M. BenChanaa, A. Mokhlisse, et A. Belghit, Energy Conversion and Management 42 (2001) $467 \mathrm{pp}$.

[15] - S. Bakkali, Afrique SCIENCE, 02(1) (2006) 116-130.

[16] - N. Bezzi, D. Merabet, N. Benabdeslem, et H. Arkoub, Ann. Chim. Sci Mat., 26 (6) (2001) 5-23.

[17] - D. Merabet, N. Benabdeslam, N. Bezzi, T. Ikhlef, H. Arkoub, Ann chim, Vol. 29, №5 (2004) 69-85. 